\title{
Optimal production planning with capacity reservation and convex capacity costs
}

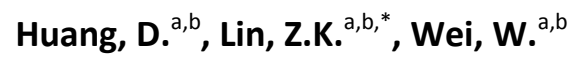 \\ ${ }^{\mathrm{a}}$ School of Economics and Management, Beijing Jiaotong University, Beijing, P.R. China \\ ${ }^{\mathrm{b}}$ Beijing Key Laboratory of Logistics Management and Technology, Beijing Jiaotong University, Beijing, P.R. China
}

\section{A B S T R A C T}

In this paper, we develop an analytical model for a multi-period production planning problem with dual supply sources of production capacity, where the supply price of one source (i.e., the spot market) is random and the supply capacity of the other source (i.e., the contract supplier) is limited. The purchasing cost of reserved capacity is assumed to be a convex function, rather than a linear function. We solve this problem by first characterizing the structure of the optimal production policy by employing a stochastic dynamic programming approach, and then determining the optimal capacity reservation level by applying a single-variable optimization method. For any given level of capacity reservation, the optimal periodic production policy is a quantity-dependent base stock policy with a threshold of production quantity that increases with the spot price. With this structure of the optimal production policy, the expected total discounted cost function is shown to be convex in the capacity reservation level. These results are also extended to the infinitehorizon case. A numerical study is conducted to examine the impacts of spot market characteristics on the optimal capacity reservation level and the corresponding optimal total cost.
\end{abstract}

\section{ARTICLE INFO}

Keywords:

Production planning;

Capacity reservation

Stochastic programming;

Optimization;

Optimal policy;

Base-stock

*Corresponding author:

zklin@bjtu.edu.cn

(Lin, Z.K.)

Article history:

Received 26 December 2017

Revised 22 February 2018

Accepted 24 February 2018

(C) 2018 PEI, University of Maribor. All rights reserved.

\section{References}

[1] Snyder, L.V., Atan, Z., Peng, P., Rong, Y., Schmitt, A.J., Sinsoysal, B. (2016). OR/MS models for supply chain disruptions: A review, IIE Transactions, Vol. 48, No. 2, 89-109, doi: 10.1080/0740817X.2015.1067735.

[2] Chen, Y., Xue, W., Yang, J. (2013). Technical note - Optimal inventory policy in the presence of a long-term supplier and a spot market, Operations Research, Vol. 61, No. 1, 88-97, doi: 10.1287/opre.1120.1114.

[3] Appleinsider. Apple chip suppliers gearing up capacity for 'iPhone 7' production - report, from https://forums. appleinsider.com/discussion/191903, accessed June 17, 2017.

[4] Silver, E.A., Jain, K. (1994). Some ideas regarding reserving supplier capacity and selecting replenishment quantities in a project context, International Journal of Production Economics, Vol. 35, No. 1-3, 177-182, doi: 10.1016/ 0925-5273(94)90079-5.

[5] Jain, K., Silver, E.A. (1995). The single period procurement problem where dedicated supplier capacity can be reserved, Naval Research Logistics, Vol. 42, No. 6, 915-934, doi: 10.1002/1520-6750(199509)42:6<915::AIDNAV3220420605>3.0.CO;2-M.

[6] Costa, D., Silver, E.A. (1996). Exact and approximate algorithms for the multi-period procurement problem where dedicated supplier capacity can be reserved, OR Spectrum, Vol. 18, No. 4, 197-207, doi: 10.1007/ BF01540156.

[7] Brown, A.O., Lee, H.L. (1997). Optimal pay-to-delay capacity reservation with application to the semiconductor industry, Working paper, Department of Industrial Engineering and Engineering Management, Stanford University, USA.

[8] Peleg, B., Lee, H.L., Hausman, W.H. (2002). Short-term e-procurement strategies versus long-term contracts, Production and Operations Management, Vol. 11, No. 4, 458-479, doi: 10.1111/j.1937-5956.2002.tb00472.x. 
[9] Kleindorfer, P.R., Wu, J.D. (2003). Integrating long- and short-term contracting via business-to-business exchanges for capital-intensive industries, Management Science, Vol. 49, No. 11, 1597-1615, doi: 10.1287/mnsc. 49.11.1597.20583.

[10] Barnes-Schuster, D., Bassok, Y., Anupindi, R. (2002). Coordination and flexibility in supply contracts with options, Manufacturing \& Service Operations Management, Vol. 4, No. 3, 171-207, doi: 10.1287/msom.4.3.171.7754.

[11] Eppen, G.D., Iyer, A.V. (1997). Backup agreements in fashion buying - The value of upstream flexibility, Management Science, Vol. 43, No. 11, 1469-1484, doi: 10.1287/mnsc.43.11.1469.

[12] Tsay, A.A., Lovejoy, W.S. (1999). Quantity flexibility contracts and supply chain performance, Manufacturing \& Service Operations Management, Vol. 1, No. 2, 89-111, doi: 10.1287/msom.1.2.89.

[13] Erkoc, M., Wu, S.D. (2005). Managing high-tech capacity expansion via reservation contracts, Production and Operations Management, Vol. 14, No. 2, 232-251, doi: 10.1111/j.1937-5956.2005.tb00021.x.

[14] Jin, M., Wu, S.D. (2007). Capacity reservation contracts for high-tech industry, European Journal of Operational Research, Vol. 176, No. 3, 1659-1677, doi: 10.1016/i.ejor.2005.11.008.

[15] Pei, P.P.-E., Simchi-Levi, D., Tunca, T.I. (2011). Sourcing flexibility, spot trading, and procurement contract structure, Operations Research, Vol. 59, No. 3, 578-601, doi: 10.1287/opre.1100.0905.

[16] Serel, D.A., Dada, M., Moskowitz, H. (2001). Sourcing decisions with capacity reservation contracts, European Journal of Operational Research, Vol. 131, No. 3, 635-648, doi: 10.1016/S0377-2217(00)00106-5.

[17] Serel, D.A. (2007). Capacity reservation under supply uncertainty, Computers \& Operations Research, Vol. 34, No. 4, 1192-1220, doi: 10.1016/j.cor.2005.06.018.

[18] Inderfurth, K., Kelle, P., Kleber, R. (2013). Dual sourcing using capacity reservation and spot market: Optimal procurement policy and heuristic parameter determination, European Journal of Operational Research, Vol. 225, No. 2, 298-309, doi: 10.1016/i.ejor.2012.08.025.

[19] Inderfurth, K., Kelle, P. (2011). Capacity reservation under spot market price uncertainty, International Journal of Production Economics, Vol. 133, No. 1, 272-279, doi: 10.1016/j.ijpe.2010.04.022.

[20] Wang, C., Liu, X.-B., Zhao, G.-Z., Chin, K.O. (2014). Multi-objective integrated production planning model and simulation constrained doubly by resources and materials, International Journal of Simulation Modelling, Vol. 13, No. 2, 243-254, doi: 10.2507/IJSIMM13(2)C010.

[21] Chen, Y.X. (2016). Integrated optimization model for production planning and scheduling with logistics constraints, International Journal of Simulation Modelling, Vol. 15, No. 4, 711-720, doi: 10.2507/IJSIMM15(4)C016.

[22] Gordić, B. (2017). Flexible optimization in the process of planning and production control, Tehnički VjesnikTechnical Gazette, Vol. 24, No. 4, 1087-1094, doi: 10.17559/TV-20160112132024.

[23] Tang, M., Gong, D., Liu, S., Lu, X. (2017). Finding key factors affecting the locations of electric vehicle charging stations: A simulation and ANOVA approach, International Journal of Simulation Modelling, Vol. 16, No. 3, 541554, doi: 10.2507/IJSIMM16(3)C015.

[24] Tang, M., Qi, Y., Zhang, M. (2017). Impact of product modularity on mass customization capability: An exploratory study of contextual factors, International Journal of Information Technology \& Decision Making, Vol. 16, No. 4, 939-959, doi: 10.1142/S0219622017410012.

[25] Gong, D., Tang, M., Liu, S., Li, Q. (2017). Reconsidering production coordination: A principal-agent theory-based analysis, Advances in Production Engineering \& Management, Vol.12, No.1,51-61, doi: 10.14743/apem2017.1.239.

[26] Wen, F., He, Z., Dai, Z., Yang, X. (2014). Characteristics of investors' risk preference for stock markets, Economic Computation and Economic Cybernetics Studies and Research, Vol. 48, No. 3, 235-254.

[27] Wen, F., Gong, X., Cai, S. (2016). Forecasting the volatility of crude oil futures using HAR-type models with structural breaks, Energy Economics, Vol. 59, 400-413, doi: 10.1016/j.eneco.2016.07.014.

[28] Wen, F., Xiao, J., Huang, C., Xia, X. (2018). Interaction between oil and US dollar exchange rate: Nonlinear causality, time-varying influence and structural breaks in volatility, Applied Economics, Vol. 50, No. 3, 319-334, doi: 10.1080/00036846.2017.1321838.

[29] Zhou, M., Liu, X., Pan, B., Yang, X., Wen, F., Xia, X. (2017). Effect of tourism building investments on tourist revenues in China: A spatial panel econometric analysis, Emerging Markets Finance and Trade, Vol. 53, No. 9, 19731987, doi: 10.1080/1540496X.2016.1237353.

[30] Hu, C., Liu, X., Pan, B., Chen, B., Xia, X. (2017). Asymmetric impact of oil price shock on stock market in China: A combination analysis based on SVAR model and NARDL model, Emerging Markets Finance and Trade, Vol. 53, No. 9, 1973-1987, doi: 10.1080/1540496X.2017.1412303.

[31] Tomlin, B. (2006). On the value of mitigation and contingency strategies for managing supply chain disruption risks, Management Science, Vol. 52, No. 5, 639-657, doi: 10.1287/mnsc.1060.0515.

[32] Henig, M., Gerchak, Y., Ernst, R., Pyke, D.F. (1997). An inventory model embedded in designing a supply contract, Management Science, Vol. 43, No. 2, 184-189, doi: 10.1287/mnsc.43.2.184.

[33] Heyman, D.P., Sobel, M.J. (1984). Stochastic models in operations research (Vol. II), McGraw-Hill, New York, USA. 